\title{
Determining the Position of the Moving Persons in 3D Space by UWB Sensors using Taylor Series Based Localization Method
}

\author{
Dušan Kocur*, Mária Švecová**, Peter Kažimír* \\ * Department of Electronics and Multimedia Communications \\ ** Department of Mathematics and Theoretical Informatics \\ Faculty of Electrical Engineering and Informatics \\ Technical University of Košice \\ B. Němcovej 32, 04200 Košice, Slovak Republic \\ Dusan.Kocur@tuke.sk, Maria.Svecova@tuke.sk, petokazimir@tuke.sk
}

\begin{abstract}
The person localization by ultra-wideband (UWB) sensors is a challenging field attracting researchers worldwide. Whereas the issue of the person localization in 2dimensional space $(2 D)$ has been discussed in many articles, only a few papers have been devoted to the people localization in 3-dimensional space (3D). Combining two $3 D$ localization methods a new approach to the person localization in $3 D$ can be obtained to fill this gap. The new $3 D$ localization method introduced in this paper is referred to as the Taylor series based localization method (TSM). This method combines the 3D-2D method of object localization in $3 D$ with the conventional method of Taylor series. The performance properties of the introduced TSM will be illustrated via the experimental scenario intent on the through-the-wall localization of a moving person by a multistatic $U W B$ radar system.
\end{abstract}

Keywords: 3D localization; signal processing; target tracking; Taylor series based localization method; UWB radar

\section{Introduction}

In the last decade, a great effort has been devoted to the study of ultra-wideband (UWB) radar applications for people monitoring [1]. The analyses of performance properties of person monitoring systems based on UWB radar (sensor) have shown that such systems allow localize multiple moving and static persons, as well as persons moving with a change of the character of their motion, not only for line-of-sight (LOS) but also for non-line-of-sight (NLOS) scenarios (e.g. localization of person situated behind of non-metallic obstacle, or localization of person e.g. in rain, snowstorm, dust, smoke, etc. [2]). It is also well-known, due to 
fine range resolution based on time-of-arrival (TOA) measurement [3], UWB sensors can provide object localization with high accuracy as well. Moreover, signals transmitted by UWB radars are extremely low-power. As results, they produce only low-level interference of narrowband communication infrastructure and thus they can more easily coexist with such communication systems. These and further unique properties of UWB sensors (comprehensively summarized, e.g. in [1]) have been the impetus in the last years for relatively extensive development of applications of UWB localization systems. Rescue and security operations, critical infrastructures monitoring [1], [4], [5], [6], senior monitoring within their dwellings [7], [8], baby monitoring (e.g. detection of sudden death syndrome), etc. are just a few examples of such applications. Moreover, due to the development of communication and sensor networks, smart-home, smart-cities, Internet of Things and low-cost UWB sensor systems, it is expected the growth of requests for contactless monitoring of people in the near future.

Motivated by these findings we have focused our research on moving people monitoring (e.g. [9], [10], [11], [12], etc.) by means of UWB radars. In this area, great attention has been devoted to the localization of persons through a vertical wall in 2-dimensional (2D) and 3-dimensional space (3D) (e.g. [1], [13]) as well as through-the-floor localization of persons in 3D [10] or to the localization of persons situated behind a corner [14].

While the issue of person localization in 2D has been addressed in many articles, people localization in 3D and localization of persons situated behind a corner has been studied only in a few papers. In order to fill this gap, we prepared this paper focused on person localization through a vertical wall in 3D. The method which will be introduced in this paper is based on the estimation of the length of time interval necessary for electromagnetic waves to travel from transmitting antenna to a target and from the target to the radar receiving antenna. Note that this is time interval is commonly referred to as TOA. In addition, our method will be based also on the application of the antenna array (Fig. 1) designed in [15] containing one transmitting antenna and four receiving antennas suitable for localizing objects in $3 \mathrm{D}$.

For the object localization based on TOA measurement for a general antenna array lay-out several iterative and non-iterative algorithms were developed, e.g. in [16], [17], [18]. As an example, a few algorithm such as iterative Taylor series method [19], [20], approximate maximum likelihood methods [21], least-squares algorithms or various localization algorithms based on target bistatic range estimation (e.g. [10], [14], [15], [22]) could be mentioned.

The target localization in 3D by means of UWB radar can be simplified by the application of the antenna array outlined in Fig. 1. Due to the antenna array layout, a geometrical interpretation of the considered localization problem allows finding the target coordinates by a simple computation of 2 intersections of 2 pairs of ellipses as was suggested in [10], [15]. The mentioned ellipses are defined by the 
antenna array layout and estimated TOAs corresponding to the target and to particular pairs of transmitting antenna and receiving antenna. This approach to person localization in 3D can be interpreted as the object localization in the $x-y$ plane (i.e. localization in 2D) and in $y$ - $z$ plane (i.e. the second localization in 2D). Then, the final target coordinates can be determined by a fusion of the results of target localization in these two planes [10], [15]. The person localization in 3D based on this idea which has been developed in [10] is known as the 3D-2D method. Its simplified version introduced in [15] is sometimes referred to as approximate 3D-2D method (A-3D-2D).

3D-2D and A-3D-2D method are simple providing quite good accuracy of person localization. However, they do not exploit the full potential of antenna array containing four receiving antennas. One can expect that some improvement in terms of the localization accuracy could be achieved if some optimization principle would be combined with the 3D-2D method or A-3D-2D method. Following this idea originally outlined in [23], the Taylor series based localization method is introduced in this paper. The main novelty of this method consists in the application of the 3D-2D method or the A-3D-2D method in a combination with the well-known Taylor series localization principle. The Taylor series based localization method is here derived for the 3D localization with the respect the antenna array layout according to Fig. 1. The performance properties of the Taylor series based localization method will be examined via the experiment focused on the through-the-wall positioning of a moving person. The comparison of the true and the estimated tracks of the person will show that the Taylor series based localization method can provide better accuracy of the localization in comparison with the localization only by the 3D-2D method.

The paper is organized as follows. The problem statement concerning the localization of a person by a multistatic UWB radar is outlined in the next section. In Section 3, the Taylor series method based on the estimations of TOAs is described. Then, the iterative Taylor series algorithm is introduced. Subsequently, the performance of the Taylor series based localization method will be demonstrated in Section 5. Finally, conclusions are drawn in the last section.

\section{System Model}

Let us consider the fundamental scenario of a moving target localization by means of a multistatic UWB radar system composed of one transmitting antenna (Tx) and four receiving antennas (Rxs). To locate and track the target denoted as T, Tx emits electromagnetic waves into a monitored area, they are reflected from objects (even from T) and received by Rxs. It is assumed that the antenna configuration and the coordinates of Tx and Rxs are fixed, known, and they are denoted as $T x=$ $\left(x_{t}, y_{t}, z_{t}\right)$ and $R x_{i}=\left(x_{i}, y_{i}, z_{i}\right)$ for $i=1,2,3,4$ (Fig. 1). It is also assumed that all 
$T O A_{i}$ belonging to the $i$ th $\mathrm{Rx}$ for $i=1,2,3,4$ have been estimated for a slow-time instant $\tau$ as well. The estimated $T O A_{i}$ for $i=1,2,3,4$ correspond to the measured round trip propagation times of the electromagnetic wave transmitted by $\mathrm{Tx}$, reflected from $\mathrm{T}$ and received by the $i$ th $\mathrm{Rx}$.

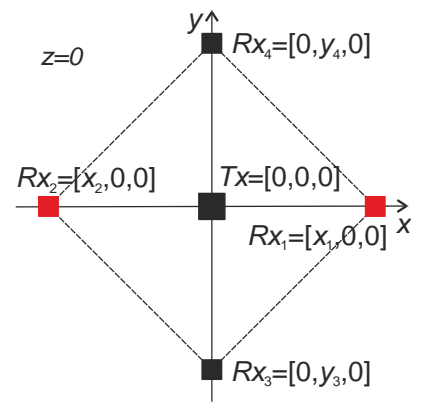

Figure 1

The antenna array layout

The total length of the trajectory along which the electromagnetic wave propagates from $\mathrm{Tx}$ to $\mathrm{T}$ and then from $\mathrm{T}$ to the $i$ th $\mathrm{Rx}$ denoted as $d_{i}$ can be expressed as a set

$d_{i}=c \cdot T O A_{i}, \quad i=1,2,3,4$,

where $c=3 \times 10^{8} \mathrm{~ms}^{-1}$ is electromagnetic wave propagation velocity in the air.

Under real conditions, the distances $d_{i}$ for $i=1,2,3,4$ are estimated with an error represented by the additive noise components $e_{i}$. Let the unknown target coordinates are denoted as $T=(x, y, z)$. Then, the estimated distances $d i$ can be modeled by the following set of the algebraic equations

$$
\begin{aligned}
& d_{i}=\|T x T\|+\left\|T R x_{i}\right\|+e_{i}= \\
& \quad \sqrt{\left(x-x_{t}\right)^{2}+\left(y-y_{t}\right)^{2}+\left(z-z_{t}\right)^{2}}+\sqrt{\left(x-x_{i}\right)^{2}+\left(y-y_{i}\right)^{2}+\left(z-z_{i}\right)^{2}}+e_{i}, \\
& i=1,2,3,4 .
\end{aligned}
$$

In these equations, $\|T x T\|$ and $\left\|T R x_{i}\right\|$ are the Euclidean distances between the points $T x$ and $T$ and between the points $T$ and $R x_{i}$, respectively. Note, that $e_{i}$ for $i=1,2,3,4$ correspond to the estimation errors of the length of the trajectory $T x-T$ $R x_{i}$. The problem of the target locating lies in determining the unknown target coordinates $(x, y, z)$ by the solution of the set of the nonlinear equations (2).

It is well known from geometry that the $i$ th equation of (2) is the equation of the spheroid obtained by rotating the ellipse about one of its principal axes. This detailed description and the corresponding mathematical formulas is beyond this paper. Its comprehensive description can be found, e.g. in [10], [24]. 
The accuracy of distance estimation $d_{i}$ is affected by several phenomena (e.g., noise, multipath, non-line-of-sight (NLOS) conditions). If the distance estimation error is additive, this results that the spheroids will not intersect at one single point. On the other hand, if the distance estimation error is subtractive, the spheroids may not intersect. So, the goal of the localization algorithm is to estimate the target position as close as the true target position, even in the presence of noisy measurements. It can be done also by the Taylor series method.

\section{Taylor Series Method}

Finding the location of the target requires the solving of the set of the nonlinear equations (2) based on TOA measurements. The most straightforward method of target position estimation is to solve a set of simultaneous equations (2). Various iterative and non-iterative position estimation algorithms have been developed [18], [22]. Non-iterative algorithms are simple and easy to implement compared to the iterative algorithms, such as spherical interpolation and other least-squares related techniques. The iterative algorithms are more complex and stop only when some pre-defined criterion is satisfied. These include, e.g. the Taylor series method.

The target localization by the nonlinear least-squares method based on a firstorder Taylor expansion (i.e. Taylor series method) was originally proposed for target locating issued from one way propagation time measurements [19]. Then, for example in [17], [26], the original Taylor series method was modified for the target localization based on the estimations of TOAs from $N$ receiving antennas. The Taylor series method based on the TOA estimations (TS) applied for the target localization in 3D according to the system model described in Section 2 can be described as follows. Here, the set of the nonlinear equations (2) is linearized by expanding it in a Taylor series around an initial estimate of a point. Only terms below second order are retained. This set of equations is solved to produce a new approximate position of the target, and the process is repeated until the stopping criterion is satisfied.

Let us define new functions of three variables $x, y, z$

$$
\begin{aligned}
f_{i}(x, y, z)= & \sqrt{\left(x-x_{t}\right)^{2}+\left(y-y_{t}\right)^{2}+\left(z-z_{t}\right)^{2}}+ \\
& \sqrt{\left(x-x_{i}\right)^{2}+\left(y-y_{i}\right)^{2}+\left(z-z_{i}\right)^{2}}, \quad i=1,2,3,4 .
\end{aligned}
$$

Then, the equations (3) can be rewritten by equations (2) as

$$
f_{i}(x, y, z)=d_{i}-e_{i}, \quad i=1,2,3,4 \text {. }
$$


Here, $e_{i}$ are the estimation errors of the distances $d_{i}$. If $x_{0}, y_{0}$, and $z_{0}$ are initial estimates of the target coordinates, which can be obtained roughly by other methods, then

$x=x_{0}+\delta_{x}, \quad y=y_{0}+\delta_{y}, \quad z=z_{0}+\delta_{z}$,

where $\delta_{x}, \delta_{y}$, and $\delta_{z}$ are the corresponding location errors to be determined. According to the selected initial coordinates $\left[\begin{array}{lll}x_{0} & y_{0} & z_{0}\end{array}\right]^{\mathrm{T}}$, expanding $f_{i}$ in a Taylor series and retaining the first two terms we can get

$f_{i o}+a_{i 1} \delta_{x}+a_{i 2} \delta_{y}+a_{i 3} \delta_{z} \approx d_{i}-e_{i}, \quad i=1,2,3,4$,

where

$$
\begin{aligned}
& f_{i o}=f_{i}\left(x_{0}, y_{0}, z_{0}\right), \\
& a_{i 1}=\frac{\partial f_{i}}{\partial x} \mid x_{0}, y_{0}, z_{0}=\frac{x_{0}-x_{t}}{r_{t 0}}+\frac{x_{0}-x_{i}}{r_{i 0}}, \\
& a_{i 2}=\frac{\partial f_{i}}{\partial y} \mid x_{0}, y_{0}, z_{0}=\frac{y_{0}-y_{t}}{r_{t 0}}+\frac{y_{0}-y_{i}}{r_{i 0}}, \\
& a_{i 3}=\frac{\partial f_{i}}{\partial z} \mid x_{0}, y_{0}, z_{0}=\frac{z_{0}-z_{t}}{r_{t 0}}+\frac{z_{0}-z_{i}}{r_{i 0}} \\
& \mathrm{r}_{t 0}=\sqrt{\left(x-x_{t}\right)^{2}+\left(y-y_{t}\right)^{2}+\left(z-z_{t}\right)^{2}} \\
& \mathrm{r}_{i 0}=\sqrt{\left(x-x_{i}\right)^{2}+\left(y-y_{i}\right)^{2}+\left(z-z_{i}\right)^{2}}
\end{aligned}
$$

Equation (6) can be rewritten in the matrix form as

$$
A \delta=D-e,
$$

where

$$
A=\left[\begin{array}{lll}
a_{11} & a_{12} & a_{13} \\
a_{21} & a_{22} & a_{23} \\
a_{31} & a_{32} & a_{33} \\
a_{41} & a_{42} & a_{43}
\end{array}\right], \delta=\left[\begin{array}{l}
\delta_{x} \\
\delta_{y} \\
\delta_{z}
\end{array}\right], D=\left[\begin{array}{l}
d_{1}-f_{10} \\
d_{2}-f_{20} \\
d_{3}-f_{30} \\
d_{4}-f_{40}
\end{array}\right], e=\left[\begin{array}{l}
e_{1} \\
e_{2} \\
e_{3} \\
e_{4}
\end{array}\right] .
$$

From equation (7) by using the least-square algorithm, the least-square solution of $\delta$ is

$$
\delta=\left[A^{T} A\right]^{-1} A^{T} \mathrm{D} .
$$

Given the initial estimations of the target coordinates $\left[\begin{array}{lll}x_{0} & y_{0} & z_{0}\end{array}\right]^{\mathrm{T}}$, the unknown values of location errors $\delta$ can be determined by (8). Then the estimations of the target coordinates are updated according to the expression 


$$
\left[\begin{array}{lll}
x_{0} & y_{0} & z_{0}
\end{array}\right]^{T}=\left[\begin{array}{lll}
x_{0}+\delta_{x} & y_{0}+\delta_{y} & z_{0}+\delta_{z}
\end{array}\right]^{T} .
$$

The computation of the target coordinates $\left[\begin{array}{lll}x_{0} & y_{0} & z_{0}\end{array}\right]^{\mathrm{T}}$ according to (9) is repeated until $\|\delta\|$ is sufficiently small, i.e. $\|\delta\|<\varepsilon$, where $\|\delta\|=\sqrt{\delta_{x}^{2}+\delta_{y}^{2}+\delta_{z}^{2}}$ denotes Euclidean norm of $\delta$ and $\varepsilon$ is the small positive number.

\section{Taylor Series Algorithm}

In the previous section, a basic principle of TS has been described. To reduce the errors of the estimated target positions emergent by the noise measurements of TOAs, TS can be considered as an iterative method of solution of (2). Then, the target localization employing TS can be implemented by using the following iteration algorithm referred to as the Taylor series algorithm:

\section{Step 0:}

To solve the equation group (2), Taylor series algorithm requires an initial target position. In this step, a reference estimate of the target coordinates denoted as $T_{0}=$ $\left(x_{0}, y_{0}, z_{0}\right)$ is obtained by using the 3D-2D method or the A-3D-2D method [15]. Note, that it is not a priori known if TOAs are or are not perfectly estimated.

\section{Step n:}

In this step, the $n$th iteration of the solution of equations (2) is done for $n=1,2$, $\ldots, N$.

The unknown coordinates of the person (target) in the $n$th iteration are estimated from the non-linear equations (2) that are linearized by the Taylor series expansion around the point $T_{n-1}=\left(x_{n-1}, y_{n-1}, z_{n-1}\right)$. The point $T_{n-1}=\left(x_{n-1}, y_{n-1}, z_{n-1}\right)$ is the estimate of the person position in the $(n-1)$ th iteration.

The group of the linear equations written in the matrix form (7) is repeatedly solved by the least-squares method at each iteration to refind a new estimate of the target coordinates denoted as $T_{n}=\left(x_{n}, y_{n}, z_{n}\right)$ by equation (8). For the updated estimate of the person position its new coordinates are refined according to $x_{n} \leftarrow x_{n-1}+\delta_{x, n}, y_{n} \leftarrow y_{n-1}+\delta_{\mathrm{y}, n}$, and $z_{n} \leftarrow z_{n-1}+\delta_{\mathrm{z}, n}$.

The parameter $\delta_{n}=\left[\delta_{x, n} \delta_{y, n} \delta_{z, n}\right]^{T}$ express a localization error in the $n$th iteration. It is obtained as the least-squares solution of (7) by (8). Here, it is used to control the iteration process of solution (2) as follows:

a) If the Euclidean norms fulfill the condition $\left\|\delta_{n-1}\right\| \leq\left\|\delta_{n}\right\|$, the iteration process is divergent. The iterative process ends and the final person position in the slow-time instant $\tau$ is $T=T_{n-1}=\left(x_{n-1}, y_{n-1}, z_{n-1}\right)$. 
b) If the Euclidean norms fulfill the conditions $\left\|\delta_{n-1}\right\|>\left\|\delta_{n}\right\|$ and $\left\|\delta_{n}\right\|>\varepsilon$, the iterating process is convergent. Note, $\varepsilon$ is a small positive number controlling the iteration process. Then, the looking for the solution of (2) will continue by the $(n+1)$ th iteration.

c) If inequations $\left\|\delta_{n-1}\right\|>\left\|\delta_{n}\right\|$ and $\left\|\delta_{n}\right\| \leq \mathcal{\varepsilon}$ are fulfilled, the iterating process ends. The final person coordinates in the slow-time instant $\tau$ are $T=T_{n}=\left(x_{n}, y_{n}, z_{n}\right)$.

d) Alternatively to (c), the iteration process ends if $n=N$, where $N$ is the preset maximum allowed number of iteration. Then, the final estimation of person positions in the slow-time instant $\tau$ is $T=T_{N}=\left(x_{N}, y_{N}, z_{N}\right)$.

The iteration algorithm of the solution of the system model (2) described herein will be referred to the Taylor series based localization method (TSM).

\section{Experimental Results}

To illustrate and validate performance properties of TSM, a scenario of the moving person localization in $3 \mathrm{D}$ (in the next referred to as the experimental scenario) will be analyzed. This scenario is outlined in Fig. 2. It is focused on the through-the-wall tracking of a person moving behind the brick wall with a thickness of $0.2 \mathrm{~m}$. The person to be localized was walking on the stairs (Fig. 3) at an approximately constant speed from the position $\mathrm{P} 1$, through the positions $\mathrm{P} 2$, P3, P4, P5, P6, P7, up to the position P8 (Fig. 2).

Table 1

The basic parameters of the UWB radar system

\begin{tabular}{|l|l|}
\hline Radar type & M-sequence UWB radar \\
\hline M-sequence order & 12 \\
\hline System clock frequency & $7 \mathrm{GHz}$ \\
\hline Operation bandwidth & $\mathrm{DC}-3 \mathrm{GHz}$ \\
\hline Length of impulse responses & $4095 \mathrm{samples}$ spread over $585 \mathrm{~ns}$ \\
\hline Radar range resolution & $0.01 \mathrm{~m}$ \\
\hline Unambiguous range & $137 \mathrm{~m}$ \\
\hline Transmitted power & $1 \mathrm{~mW}$ \\
\hline Radar configuration & One Tx and four Rxs \\
\hline Antenna spacing & $0.39 \mathrm{~m}$ \\
\hline Antenna type & Horn open \\
\hline
\end{tabular}

The person was monitored by the M-sequence UWB radar system [1] equipped with one Tx and four Rxs depicted in Fig. 4. The parameters of the radar are stated in Table 1. The five double-ridged horn antennas have been placed on the wall 
forming a wall-to-air interface. The antenna array layout is shown in Fig. 1. The distance between Tx and Rxs has been $0.39 \mathrm{~m}$, Tx has been located in the middle between four Rxs. For the target positioning in 2D, the antenna array with an appropriate layout of one Tx and two Rxs is sufficient. The antenna array consisting of $\mathrm{Tx}, \mathrm{Rx}_{1}$, and $\mathrm{Rx}_{2}$ has been located on the $x$-axis Such an antenna array should be enough for the localization of a target in the $x-y$ plane (in 2D). Similarly, the antenna array consisting of $\mathrm{Tx}, \mathrm{Rx}_{3}$ and $\mathrm{Rx}_{4}$ located on the axis $y$ should be enough for the localization of a target in the $y$ plane (in 2D). The antenna array consisting of Tx and four Rxs arranged according to Fig. 1 is proper for 3D localization.

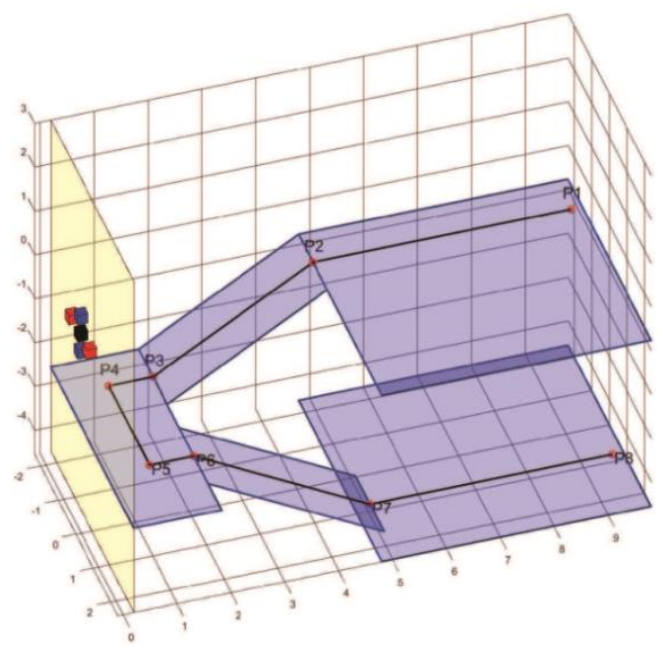

Figure 2

The scheme of measurement with the positions of antennas and the reference positions

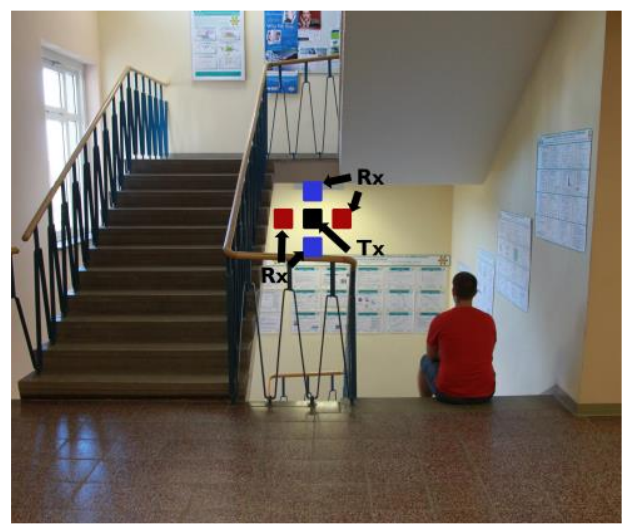

Figure 3

The monitored area

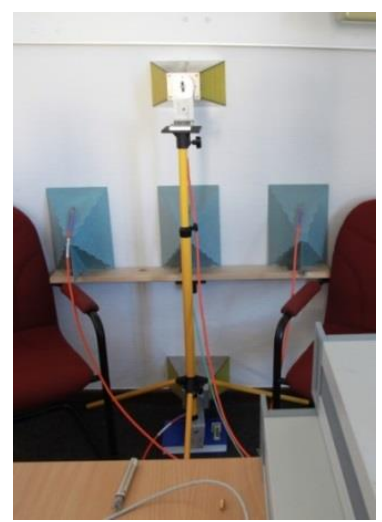

Figure 4

The measurement apparatus, M-sequence UWB radar system located behind the wall 
To locate and track a moving target, Tx emitted electromagnetic waves into a monitored area, they were reflected from objects (even from the target) and received by Rxs. The raw radar signals retrieved from the radar system can be interpreted as a set of impulse responses of the surrounding through which the electromagnetic waves spread. The set of impulse responses is referred to as radargram [1], [13]. Then, the target positions and its track in the monitored area can be obtained by the sequential processing of particular impulse responses of radargram.

The processing of the radar signals for moving person localization [13], [24], consists of the set of phases responsible for the elimination of stationary clutter (methods of background subtraction), the decision about the target presenc or absence (methods of detection), the estimation and association of distances from the same target (methods of TOA estimation), the wall effect compensation (if target detection and tracking by UWB radar is realized through the walls with known thickness and relative permittivity of the wall), the estimation of target positions (methods of localization) and finally the monitoring of target motion over time (methods of tracking). Here, the exponential averaging method [27], CFAR detector [28], [29], trace connection method [11], trace correction of the first kind [30], the 3D-2D method [15], and multiple target tracking system (MTT) [13], [27], [31] have been applied for the background subtraction, target detection, TOA estimation, wall effect compensation, target localization, and target tracking, respectively. The detailed description of the particular phases of radar signal processing together with the corresponding mathematical formulas is beyond this paper. Its comprehensive description can be found, e.g. in [13], [24].

By using the above-mentioned procedure for radar signal processing, the TOAs corresponding the target have been obtained for every slow-time instant $\tau$. Then, the positions of the target in the monitored area have been determined by the 3D2D method. The obtained results of the localization phase are depicted in Fig. 5 . These by the 3D-2D method given target coordinates have been used as the initial estimates for the target positioning by TSM. Finally, the target coordinates have been refound by TSM. By the TSM achieved results are depicted in Fig. 7. The projections of the estimated person coordinates into the $x-y$ plane are illustrated in Fig. 6 and 8.

The last phase of the radar signal processing procedure applied to target positions estimated by the localization method is target tracking. It allows improving the robustness and precision of the target coordinates estimations based on its foregoing positions. Here, the target tracking by MTT has been employed. The person tracks estimated by MTT for the 3D-2D method and TSM are depicted in Fig. 9 and 11, respectively. The projections of the estimated positions of the person into the $x-y$ plane are illustrated in Fig. 10 and 12, respectively.

Since the real width of the person is non-zero and the resolution of the radar used for measurement is approximately $0.01 \mathrm{~m}$, the alternative form of the visualization 
of the target position estimation accuracy is shown in Fig. 5-12. In these figures, the yellow cuboids and parallelepipeds, or rectangles in the projection into the $x-y$ plane, are outlined. The part of the monitored area bordered by these shapes, where the true track of the person is located, can be referred to as a region of the true positions of the person. The width of this region is set to $1 \mathrm{~m}$, its height is set to $1.8 \mathrm{~m}$. It corresponds approximately to the effective size of a human body.

The analyses of the results presented in Fig. $9-12$ show, that the estimated tracks have kept the direction of the target movement very-well. The tracks estimated by TSM and MTT is better than that of the trajectory estimated by MTT coming out of the positions estimated by the 3D-2D method. This improvement is observable especially in these segments of the tracks in which the person was changing the direction of their motion. This performance of TSM over the 3D-2D method is reached at the cost of its higher complexity in comparison with the 3D-2D method.

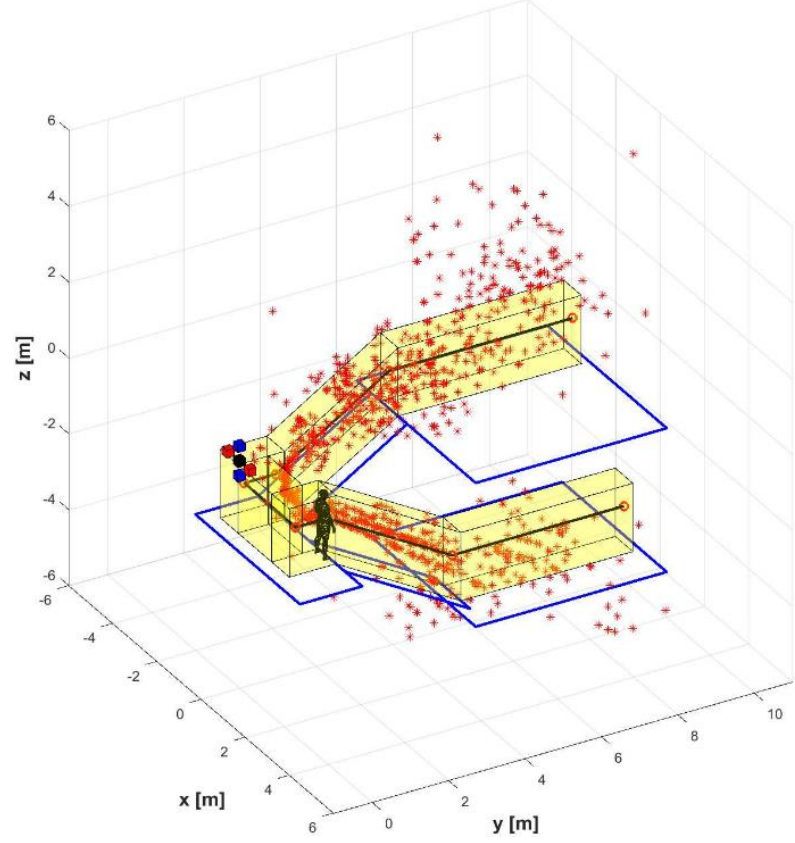

Figure 5

Person positions estimated by the 3D-2D method 


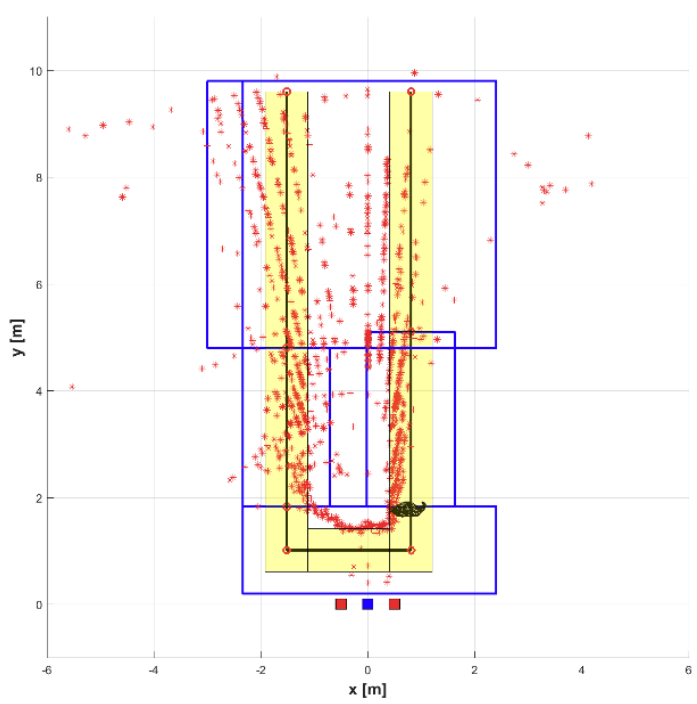

Figure 6

Person positions estimated by the 3D-2D method: the projection into $x-y$ plane

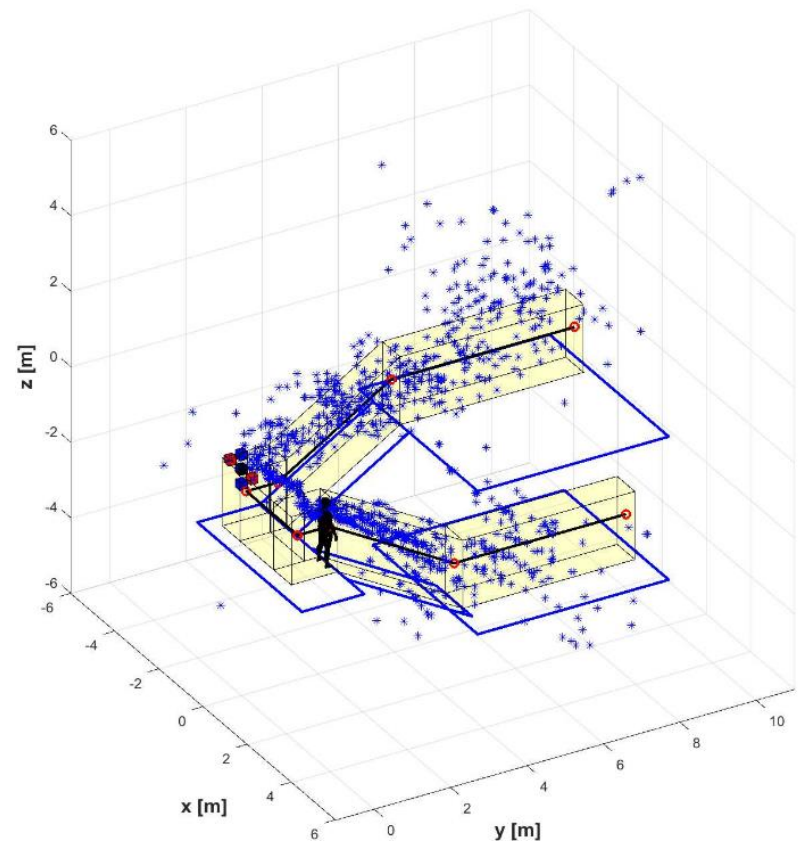

Figure 7

Person positions estimated by TSM 


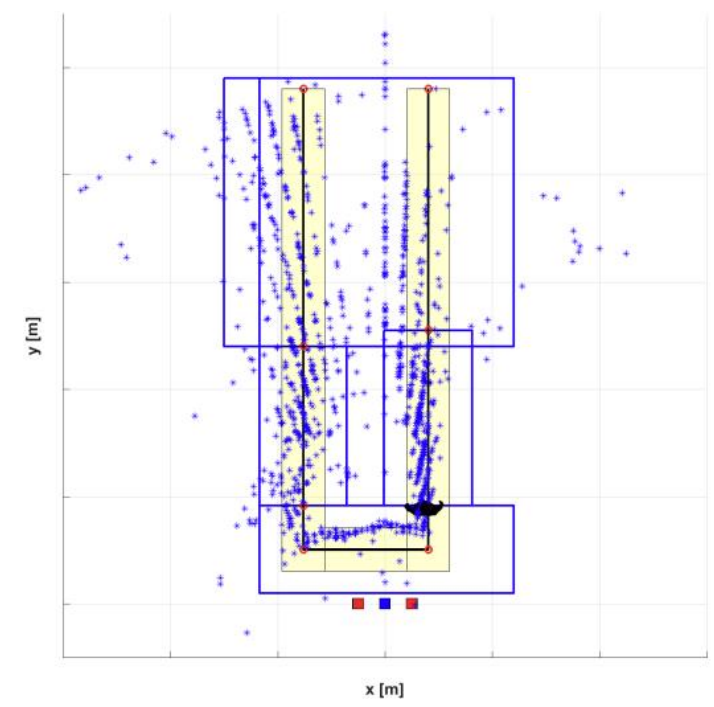

Figure 8

Person positions estimated by TSM: the projection into $x-y$ plane

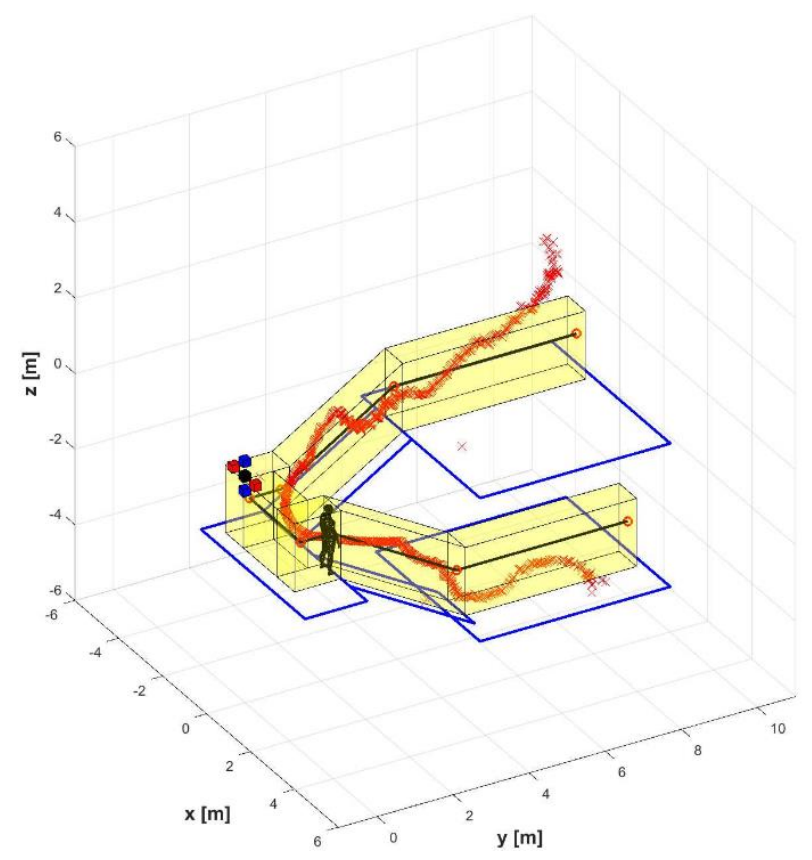

Figure 9

Person track estimated by the 3D-2D method and MTT 


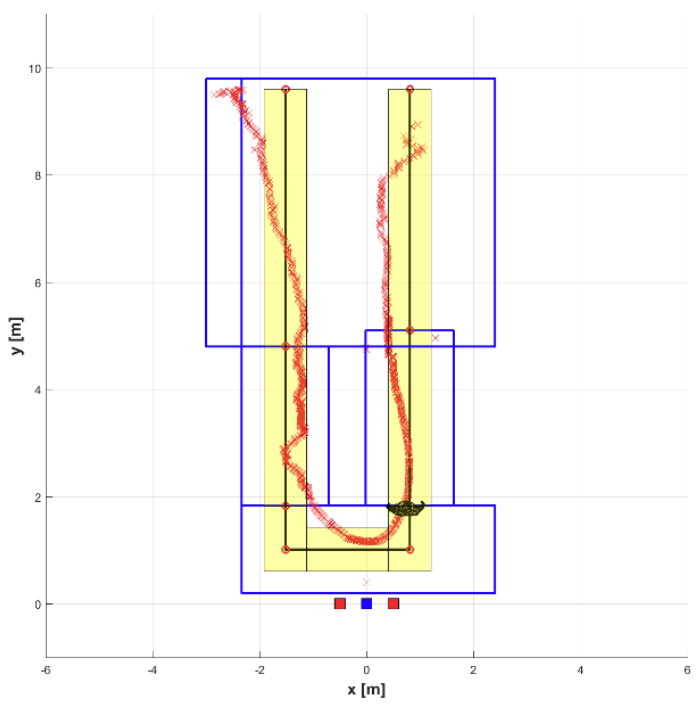

Figure 10

Person track estimated by the 3D-2D method and MTT: the projection into $x-y$ plane

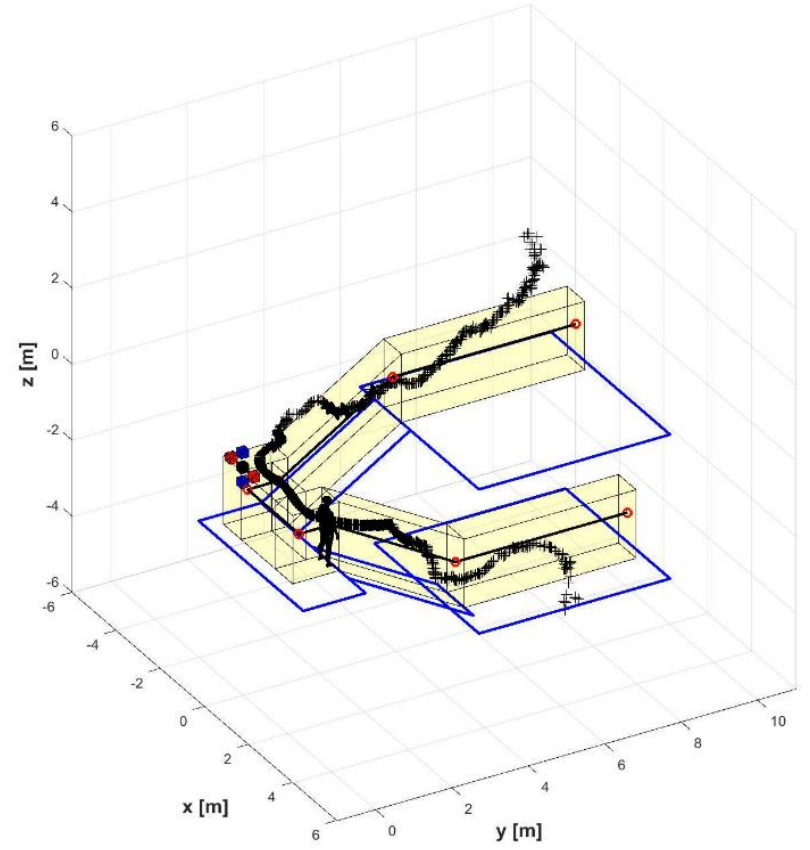

Figure 11

Person track estimated by TSM and MTT 


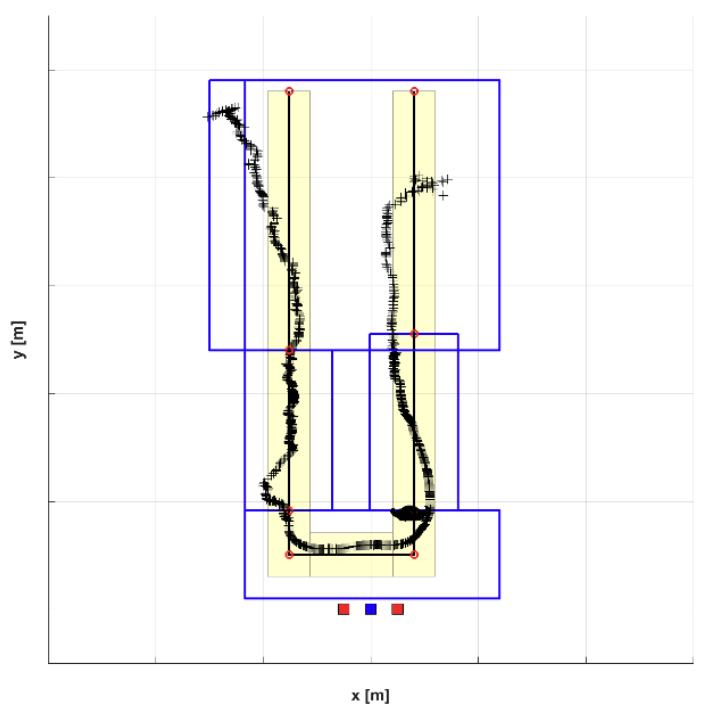

Figure 12

Person track estimated by TSM and MTT: the projection into $x-y$ plane

\section{Conclusions}

In this article, the problem of the 3D localization of a moving person using Msequence UWB radar for NLOS scenario has been studied. As has been shown in [10] and [15], for the moving person localization in 3D, the 3D-2D method [10] or its approximation [15] can be used. The advantage of these approaches consists on one hand in the simplicity of the mathematical solution of (2), and on the other hand, what is perhaps more interesting that all methods developed for the target localization in 2D can be applied for the target localization in 3D. Unfortunately, $3 \mathrm{D}-2 \mathrm{D}$ method and its approximation are capable of providing only a very rough approximation of the solution of (2). This finding including experimental experiences has motivated us to connect the 3D-2D method with a well-known iterative TS method [16]. In this concept, the 3D-2D method is used for the estimation of an initial solution of (2), and the TS method can provide an additional improvement of the target localization accuracy. As a result, the TS algorithm has been introduced in this paper as a novel approach to person localization in 3D. Moreover, the accuracy of person localization in 3D by the TS algorithm has been further improved by the implementation of target tracking in 3D. The experimental results obtained for through-the-wall localization of a single moving person has confirmed our assumptions that the TS algorithm can provide the reliable estimations of the person positions even in a complex environment. Therefore, we believe that the TS algorithm could be an interesting and successful member of the family of the algorithms to be applied for the person monitoring in 3D using UWB sensors. 
In our opinion, the TS method and TS algorithms represent a universal approach to object localization in 3D based on TOA measurement. Therefore, they can be applied not only for person monitoring but also for localization of other moving objects. The state-of-the-art in the field of localization algorithm exploitation indicates that, e.g. the field of robotics could be considered the very perspective from this point of view. The localization method could be employed, e.g. for monitoring a robot environment (e.g. [32]) or for monitoring robot itself (e.g. [33]). We also believe, the method developed in this paper could be applied, e.g. in the field of robot posing or for the solution of a problem of simultaneous localization and mapping (SLAM), where the robot must simultaneously map the environment and locate itself (e.g. [34], [35], [35], [37]).

\section{Acknowledgement}

This work was supported by the Scientific Grant Agency (VEGA) under the contract No. 1/0772/17 and by the Slovak Research and Development Agency under the contract No. APVV-18-0373.

\section{References}

[1] J. Sachs, Handbook of Ultra-wideband Short-Range Sensing: Theory, Sensors, Applications, John Wiley \& Sons, 2012

[2] B. Yamauchi, "All-weather perception for man-portable robots using ultrawideband radar," in 2010 IEEE International Conference on Robotics and Automation, Anchorage, AK, USA, 2010

[3] J. Sachs et al., "On the Range Precision of UWB Radar Sensors," in 11-th International Radar Symposium, Vilnius, Lithuania, 2010

[4] P. Withington, H. Fluhler, S. Nag, "Enhancing Homeland Security with Advanced UWB Sensors," IEEE Microwave Magazine, Vol. 4, No. 3, pp. 5158, Sept. 2003

[5] C. Huffman, L. Ericson, "Though-the-Wall Sensors (TTWS) for Law Enforcement: Market Survey,” ManTech Advanced Systems International, Inc., 2012

[6] ReTWis, „Look behind the walls,“ [Online] Available: http://www.lokalizacni-systemy.cz/en/retwis-en/

[7] J. Sachs et al., "Remote vital sign detection for rescue, security, and medical care by ultra-wideband pseudo-noise radar," Ad Hoc Networks, Vol. 13, pp. 42-53, Feb 2014

[8] G. Diraco, A. Leone, P. Siciliano, "A Radar-Based Smart Sensor for Unobtrusive Elderly Monitoring in Ambient Assisted Living Applications," Biosensors (Basel), Vol. 7, No. 4, Nov. 2017 
[9] D. Kocur, M. Švecová, D. Novák, "UWB Radar Based Localization of a Person Changing the Nature of Their Motion State," in International Conference on Radar (RADAR), Brisbane, QLD, Australia, 2018

[10] M. Švecová et al., "Through-the-floor localization of a static person by a multistatic UWB radar," Microwave and Optical Technology Letters, Vol. 61, No. 3, pp. 825-831, 2018

[11] J. Rovňáková, D. Kocur, "TOA Estimation and Data Association for Through-Wall Tracking of Moving Targets," EURASIP Journal on Wireless Communications and Networking, Vol. 2010, No. 1, p. 420767, Aug 2010

[12] D. Kocur, M. Švecová, R. Zetik, "Basic signal processing principles for monitoring of persons using UWB sensors - An overview," Acta Electrotechnica et Informatica, in press

[13] D. Kocur, J. Rovňáková, M. Švecová, “Through Wall Tracking of Moving Targets by M-Sequence UWB Radar," in Studies in Computational Intelligence, Vol. 243, Berlin, Heidelberg, Springer, 2009, pp. 349-364

[14] G. Shen et al., "Range-Based Localization for UWB Sensor Networks in Realistic Environments," EURASIP Journal on Wireless Communications and Networking, Vol. 2010, No. 1, p. 476598, Oct 2009

[15] P. Kažimír et al., "Localisation of motionless persons in 3D space by UWB radar," in PIERS Proceedings, Aug 2014

[16] K. Yu et al., "UWB location and tracking for wireless embedded networks," Signal Processing, Vol. 86, No. 9, pp. 2153-2171, Sept 2006

[17] M. Svecova, D. Kocur, R. Zetik, "Object localization using round trip propagation time measurements," in $18^{\text {th }}$ International Conference Radioelektronika, Prague, Czech Republic, April 2008

[18] A. G. Ferreira et al., "Performance Analysis of ToA-Based Positioning Algorithms for Static and Dynamic Targets with Low Ranging Measurements," Sensors (Basel), Vol. 17, No. 8, p. 1915, 2017

[19] W. H. Foy, "Position-Location Solutions by Taylor-Series Estimation," IEEE Transactions on Aerospace and Electronic Systems, Vols. AES-12, No. 2, pp. 187-194, 1976

[20] J. Ren, J. Chen, W. Bai, “A New Localization Algorithm Based on Taylor Series Expansion for NLOS Environment," Cybernetics and Information Technologies, Vol. 16, No. 5, pp. 127-136, 2016 
[21] Y.-T. Chan, H. Y. C. Hang, P.-C. Ching, "Exact and approximate maximum likelihood localization algorithms," IEEE Transactions on Vehicular Technology, Vol. 55, No. 1, pp. 10-16, 2006

[22] H. Zhang et al., "An improved Taylor series based location algorithm for IEEE 802.15.4a channel," in Proceedings of 2011 IEEE Pacific Rim Conference on Communications, Computers and Signal Processing, Victoria, BC, Canada, Aug. 2011

[23] D. Kocur, M. Švecová, P. Kažimír, "Taylor Series Based Localization Method of Moving Persons in 3D Space by UWB Sensors," in IEEE $23^{\text {rd }}$ International Conference on Intelligent Engineering Systems, Gödöllö, Hungary, April 2019

[24] D. Kocur, M. Švecová, J. Rovňáková, "Through-the-wall localization of a moving target by two independent ultra wideband (UWB) radar systems," Sensors (Bazel, Switzerland), Vol. 13, No. 9, pp. 11969-11997, 2013

[25] X. Zheng et al., "Wireless localization based on the time sum of arrival and Taylor expansion," in $19^{\text {th }}$ IEEE International Conference on Networks (ICON), Singapore, Dec 2013

[26] J. Rovňáková, Complete signal processing for through wall tracking of moving targets, LAP LAMBERT Academic Publishing, 2010

[27] H. Rohling, "Radar CFAR Thresholding in Clutter and Multiple Target Situations," IEEE Transactions on Aerospace and Electronic Systems, Vols. AES-19, No. 4, pp. 608-621, 1983

[28] G. Minkler, J. Minkler, CFAR: The Principles of Automatic Radar Detection in Clutter, Baltimore: Magellan Book Company, 1990

[29] J. Rovnakova and D. Kocur, "Compensation of wall effect for through wall tracking of moving targets," Radioengineering, Vol. 18, No. 2, pp. 189-195, 2009

[30] M. S. Grewal, A. P. Andrews, Kalman filtering: theory and practice, Upper Saddle River, NJ, USA: Prentice-Hall, Inc., 1993

[31] D. Kocur et al., "Short-range UWB radar: Surveillance robot equipment of the future," in IEEE International Conference on Systems, Man, and Cybernetics (SMC), San Diego, CA, USA, Oct 2014

[32] P. Galajda et al., "Wireless UWB Sensor System for Robot Gripper Monitoring in Non-cooperative Environments," in Recent Advances in Intelligent Engineering. Topics in Intelligent Engineering and Informatics, Springer, Cham, March 2019, pp. 177-207 
[33] Á. Takács et al., "Models for Force Control in Telesurgical Robot Systems," Acta Polytechnica Hungarica, Vol. 12, No. 8, pp. 95-114, January 2015

[34] B. Fernandez-Gauna et al., "Basic Results and Experiments on Robotic Multi-Agent System for Hose Deployment and Transportation," International Journal of Artificial Intelligence, Vol. 6, No. S11, pp. 183-202, March 2011

[35] B. Sarkar, P. K. Pal, D. Sarkar, "Building maps of indoor environments by merging line segments extracted from registered laser range scans," Robotics and Autonomous Systems, Vol. 62, No. 4, pp. 603-615, 2014

[36] P. Merriaux et al., "Robust robot localization in a complex oil and gas industrial environment," Journal of Field Robotics, Vol. 62, No. 4, pp. 213230, 2018

[37] E. Zaikov, J. Sachs, "UWB radar for detection and localization of trapped people," in Ultra Wideband, Rijeka, IntechOpen, 2010 